\title{
Verifying Average Dwell Time by Solving Optimization Problems ${ }^{\star}$
}

\author{
Sayan Mitra ${ }^{1}$, Nancy Lynch ${ }^{1}$, and Daniel Liberzon ${ }^{2}$ \\ 1 Computer Science and AI Laboratory, \\ Massachusetts Inst. of Technology, \\ 32 Vassar Street, Cambridge, MA 02139 \\ \{lynch, mitras\}@csail.mit.edu, \\ 2 Coordinated Science Laboratory, \\ Univ. of Illinois at Urbana-Champaign, \\ Urbana, IL 61821 \\ liberzon@uiuc.edu
}

\begin{abstract}
In the switched system model, discrete mechanisms of a hybrid system are abstracted away in terms of an exogenous switching signal which brings about the mode switches. The Average Dwell time $(A D T)$ property defines restricted classes of switching signals which provide sufficient conditions for proving stability of switched systems. In this paper, we use a specialization of the Hybrid I/O Automaton model to capture both the discrete and the continuous mechanisms of hybrid systems. Based on this model, we develop methods for automatically verifying ADT properties and present simulation relations for establishing equivalence of hybrid systems with respect to ADT. Given a candidate ADT for a hybrid system, we formulate an optimization problem; a solution of this problem either establishes the ADT property or gives an execution fragment of the system that violates it. For two special classes of hybrid systems, we show that the corresponding optimization problems can be solved using standard mathematical programming techniques. We formally define equivalence of two hybrid systems with respect to ADT and present a simulation relation-based method for proving this equivalence. The proposed methods are applied to verify ADT properties of a linear hysteresis switch and a nondeterministic thermostat.
\end{abstract}

\section{Introduction}

In order to accurately represent hybrid phenomena that arise in typical applications, hybrid system models must provide discrete and continuous valued state variables and must have mechanisms to capture both instantaneous state transitions and state trajectories spanning time intervals. The standard approach for describing hybrid behavior is to assume that every state of the system belongs to one of $\mathcal{P}$ modes, where $\mathcal{P}$ is a finite index set. When the state is in mode $p$, for some $p \in \mathcal{P}$, the continuous variables $\mathbf{x}$ evolve according to $\dot{\mathbf{x}}=f_{p}(\mathbf{x})$ and

\footnotetext{
* Supported by the MURI project:DARPA/AFOSR MURI F49620-02-1-0325 grant.
} 
the discrete variables remain constant. Discrete transitions alter both continuous and discrete variables, which may lead to mode change. Analyzing the stability of hybrid systems is challenging because the stability of the continuous dynamics of each individual mode does not necessarily imply the stability of the whole system (see [2] for an example). The basic tool for studying stability of hybrid systems relies on the existence of a Common Lyapunov function, whose derivative along the trajectories of all the modes must satisfy suitable inequalities. When such a function is not known or does not exist, Multiple Lyapunov functions [2] are useful for proving stability of a chosen execution. These and many other stability results are based on the switched system $[9,15]$ view of hybrid systems. Switched systems may be seen as higher-level abstractions of hybrid systems. A switched system model neglects the details of the discrete behavior of a hybrid system and instead relies on an exogenous switching signal to bring about the mode switches. If the individual modes of the system are stable, then the Dwell Time [14] and the more general Average Dwell Time (ADT) criteria, introduced by Morse and Hespanha [6], define restricted classes of switching signals that guarantee stability of the whole system. In this paper, we present techniques for automatically verifying ADT properties using a model for hybrid systems that captures both their discrete and continuous mechanisms. Thus we provide a missing piece in the toolbox for analysis of stability of hybrid systems.

We use the Hybrid Input/Output Automaton (HIOA) framework of Lynch, Segala, and Vaandrager [11] to develop methods for checking ADT properties, which in turn provide sufficient conditions for proving stability of hybrid systems. A hybrid system $\mathcal{A}$ has $\operatorname{ADT} \tau_{a}$ if, in every execution fragment of $\mathcal{A}$, any $\tau_{a}$ interval of time, on an average, has at most one mode switch. A large ADT means that the system spends enough time in each mode to dissipate the transient energy gained through mode switches. Having a large ADT itself is not sufficient for stability; in addition, the individual modes of the automaton must also be stable. In fact, the problem of testing the stability of a hybrid system can be broken down into (a) finding Lyapunov functions for the individual modes and (b) checking the ADT property. We assume that a solution to part (a) a set of Lyapunov functions for the individual modes - is known from existing techniques, and we present automatic methods for part (b).

Our approach for checking if a given automaton $\mathcal{A}$ has $\operatorname{ADT} \tau_{a}$, is to formulate an optimization problem $\operatorname{OPT}\left(\tau_{a}\right)$. From the solution of $\operatorname{OPT}\left(\tau_{a}\right)$ we can either get a counterexample execution fragment of $\mathcal{A}$ that violates the ADT property $\tau_{a}$, or else we can conclude that no such counterexample exists and that $\mathcal{A}$ has $\operatorname{ADT} \tau_{a}$. We show that for certain useful classes of $\operatorname{HIOA}, \mathrm{OPT}\left(\tau_{a}\right)$ can indeed be formulated and solved using standard mathematical programming techniques. In [12], an invariant-based method for proving ADT is proposed. This method transforms the given automaton $\mathcal{A}$ to a new automaton $\mathcal{A}_{\tau_{a}}$, so that $\mathcal{A}$ has $\mathrm{ADT}$ $\tau_{a}$ if and only if $\mathcal{A}_{\tau_{a}}$ has a particular invariant property $\mathcal{I}_{\tau_{a}}$. This method is applicable to any HIOA; however, for general HIOA, the invariant $\mathcal{I}_{\tau_{a}}$ cannot be checked automatically. The optimization-based approach presented here is automatic and complements the invariant method of [12] because the two can be 
used in combination to find the ADT of hybrid systems. We can start with some candidate value of $\tau_{a}>0$ and search for a counterexample execution fragment for it, using the optimization-based approach. If such an execution fragment is found, then we decrease $\tau_{a}$ (say, by a factor of 2) and try again. If eventually the optimization approach fails to find a counterexample execution fragment for a particular value of $\tau_{a}$, then we use the invariant approach to prove that this value of $\tau_{a}$ is an ADT for the given system.

Contributions and overview. In Section 2 we introduce a new model for hybrid systems called Structured Hybrid Automaton (SHA). We define stability and the Average Dwell Time (ADT) property of SHA. In Section 3 we introduce the optimization problem $\mathrm{OPT}\left(\tau_{a}\right)$, which searches for an execution fragment of the given SHA that violates the ADT property in question. We formally define what it means for one SHA to switch faster than another and for two SHA to be ADT-equivalent. We define a new type of simulation relation, called switching simulation, that provides sufficient conditions for establishing the "faster than" and the equivalence relationships between SHA. In Section 4 we explore the class of Graph SHA, for which solving $\mathrm{OPT}\left(\tau_{a}\right)$ reduces to detecting a negative cost cycle in a weighted graph. We verify the ADT property of a linear, scaleindependent hysteresis switch taken from [5] by first finding a Graph SHA $\mathcal{B}$ that is ADT-equivalent to it and then showing how $\mathrm{OPT}\left(\tau_{a}\right)$ for $\mathcal{B}$ can be solved efficiently using standard graph algorithms, like the Bellman-Ford algorithm [3]. In Section 5, we study a more general class of SHA, called Initialized SHA and show that OPT $\left(\tau_{a}\right)$ can be solved by detecting a cyclic execution fragment with "extra" mode switches. We show that for rectangular initialized SHA, OPT $\left(\tau_{a}\right)$ can be formulated as a Mixed Integer Linear Program. We use this formulation along with switching simulations to verify the ADT property of a nondeterministic thermostat.

\section{$2 \quad$ Hybrid system model and stability definitions}

The Hybrid Input/Output Automaton (HIOA) model [11] with its invariant and simulation based proof methods has been used to verify the safety properties of several hybrid systems (see, e.g., [10,13, 4]). In this paper, we are concerned with internal stability of hybrid systems, so we use a specialization of the HIOA model called Structured Hybrid Automata (SHA), that does not have input/output variables and does not distinguish among input, output, and internal actions. On the other hand, SHA have extra structure called "state models" for describing the trajectories using differential and algebraic equations.

\subsection{Structured Hybrid Automaton model}

We denote the domain of a function $f$ by f.dom. For a set $S \subseteq f$.dom, we write $f\lceil S$ for the restriction of $f$ to $S$. If $f$ is a function whose range is a set of functions containing $Y$, then we write $f \downarrow Y$ for the function $g$ with 
g.dom $=$ f.dom such that for each $c \in$ g.dom, $g(c)=f(c)\lceil Y$. For a tuple or an array $b$ with $n$ elements, we refer to its $i^{\text {th }}$ element by $b[i]$.

We fix the time axis $\mathrm{T}$ to be $\mathrm{R}_{\geq 0}$. Let $X$ be a set of state variables; $X$ is partitioned into $X_{d}$, the set of discrete variables, and $X_{c}$, the set of continuous variables. Each variable $x \in X$ is associated with a type, which is the set of values that $x$ can assume. Each $x \in X_{d}$ (respectively, $X_{c}$ ) has dynamic type, which is the pasting closure of the set of constant (resp. continuous) functions ${ }^{3}$ from left-closed intervals in $\mathrm{T}$ to the type of $x$. A valuation $\mathrm{x}$ for the set of variables $X$ is a function that associates each $x \in X$ to a value in its type. The set of all valuations of $X$ is denoted by $\operatorname{val}(X)$. A trajectory $\tau: J \rightarrow \operatorname{val}(X)$ specifies the values of all variables $X$ on a time interval $J$ with left endpoint of $J$ equal to 0 , with the constraint that evolution of each $x \in X$ over the trajectory should be consistent with its dynamic type. A trajectory with domain $[0,0]$ is called a point trajectory. If $\tau$.dom is right closed then $\tau$ is closed and its limit time is the supremum of $\tau$.dom and is written as $\tau$.ltime. The first valuation of $\tau, \tau$.fval is $\tau(0)$, and if $\tau$ is closed, then the last valuation of $\tau, \tau . l v a l$, is $\tau(\tau$. ltime $)$.

Definition 1. A state model $F$ for a set of variables $X$ is a set of differential equations for $X_{c}$ of the form $\dot{\mathbf{x}}_{c}=f\left(\mathbf{x}_{c}\right)$, such that: (1) For every $\mathbf{x} \in \operatorname{val}(X)$, there exists a trajectory $\tau$ with $\tau$. fval $=\mathbf{x}$, with the property that $\tau \downarrow X_{c}$ satisfies $F$, and (2) for all $t \in \tau$.dom, $\left(\tau \downarrow X_{d}\right)(t)=\left(\tau \downarrow X_{d}\right)(0)$. The prefix and suffix closure of the set of trajectories of $X$ that satisfy the above conditions is denoted by $\operatorname{traj}(X, F)$.

Definition 2. A Structured Hybrid Automaton (SHA) is a tuple $\mathcal{A}=(X, Q, \Theta$,$A, \mathcal{D}, P)$, where (1) $X$ is a set of variables, including a special discrete variable called mode. (2) $Q \subseteq \operatorname{val}(X)$ is the set of states, (3) $\Theta \subseteq Q$ is a nonempty set of start states, (4) $A$ is a set of actions, (5) $\mathcal{D} \subseteq Q \times A \times Q$ is a set of discrete transitions, and (6) $P$ is an indexed family $F_{i}, i \in \mathcal{P}$, of state models, where $\mathcal{P}$ is an index set.

A transition $\left(\mathbf{x}, a, \mathbf{x}^{\prime}\right) \in \mathcal{D}$ is written in short as $\mathbf{x} \stackrel{a}{\rightarrow} \mathcal{A} \mathbf{x}^{\prime}$ or as $\mathbf{x} \stackrel{a}{\rightarrow} \mathbf{x}^{\prime}$ when $\mathcal{A}$ is clear from the context. A transition $\mathbf{x} \stackrel{a}{\rightarrow} \mathbf{x}^{\prime}$ is a mode switch if $\mathbf{x}\lceil$ mode $\neq$ $\mathbf{x}^{\prime}\lceil$ mode. The set of mode switching transitions is denoted by $M$. The guard predicate of action $a$ is $G_{a} \triangleq\left\{\mathbf{x} \in Q \mid \exists \mathbf{x}^{\prime}, \mathbf{x} \stackrel{a}{\rightarrow} \mathbf{x}^{\prime} \in \mathcal{D}\right\}$. In this paper, we assume that the right hand sides of the differential equations in the state models are well behaved (locally Lipschitz), and the differential equations have solutions defined globally in time. Therefore, for each $F_{i}, i \in \mathcal{P}$ and $\mathbf{x} \in Q$ with $\mathbf{x}\left\lceil\right.$ mode $=i$, there exists a trajectory $\tau$ starting from $\mathbf{x}$ that satisfies $F_{i}$ and if, $\tau$.dom is finite then $\tau$.lval $\in G_{a}$ for some $a \in A$. The set $\mathcal{T}$ of trajectories of SHA $\mathcal{A}$ is defined as $\mathcal{T} \triangleq \bigcup_{i \in \mathcal{P}} \operatorname{traj}\left(X, F_{i}\right)$. An execution fragment of an SHA $\mathcal{A}$ is an alternating sequence of actions and trajectories $\alpha=\tau_{0} a_{1} \tau_{1} a_{2} \ldots$, where (1) each $\tau_{i} \in \mathcal{T}$, and (2) if $\tau_{i}$ is not the last trajectory then $\tau_{i}$.lstate $\stackrel{a_{i+1}}{\rightarrow} \tau_{i+1}$.f state.

3 This set of functions must be closed under time-shift, restriction to subintervals, and pasting. See [7] for formal definition of these closure properties 
The first state of an execution fragment $\alpha, \alpha . f$ state, is $\tau_{0} . f$ state. An execution fragment $\alpha$ is an execution of $\mathcal{A}$ if $\alpha$.f state $\in \Theta$. The length of a finite execution fragment $\alpha$ is the number of actions in $\alpha$. An execution fragment is closed if it is a finite sequence, and the domain of the last trajectory is closed. Given a closed execution fragment $\alpha=\tau_{0}, a_{1}, \ldots, \tau_{n}$, its last state, $\alpha . l s t a t e$, is $\tau_{n} . l$ state and its limit time is defined as $\sum_{i}^{n} \tau_{i}$.ltime. We define the following shorthand notation for the valuation of the variables of $\mathcal{A}$ at $t \in[0, \alpha$. ltime $]: \alpha(t)=\alpha^{\prime}$.lstate, where $\alpha^{\prime}$ is the longest prefix of $\alpha$ with $\alpha^{\prime}$.ltime $=t$. A state $\mathbf{x} \in Q$ is reachable if it is the last state of some execution of $\mathcal{A}$. An execution fragment $\alpha$ is reachable if $\alpha$.f state is reachable. An invariant property or simply an invariant $\mathcal{I}$ of $\mathcal{A}$ is a condition on $X$ that remains true in all reachable states of $\mathcal{A}$. A closed execution fragment $\alpha$ of SHA $\mathcal{A}$ is a cycle if $\alpha$. fstate $=\alpha$.lstate.

\subsection{Stability and Average Dwell Time}

Stability is a property of the continuous variables of SHA $\mathcal{A}$ with respect to the standard Euclidean norm in $R^{n}$. At a given state $\mathbf{x} \in Q$, we write the norm of the continuous variables $\mid \mathbf{x}\left\lceil X_{c} \mid\right.$ in short as $|\mathbf{x}|$. We assume that for each $i \in \mathcal{P}$, the origin is an equilibrium point for the state model $\dot{\mathbf{x}}_{c}=f_{i}\left(\mathbf{x}_{c}\right)$ of $\mathcal{A}$.

SHA $\mathcal{A}$ is stable (also called stable in the sense of Lyapunov), if for every $\epsilon>0$, there exists a $\delta>0$, such that for every closed execution $\alpha$ of $\mathcal{A}$, for all $t \in[0, \alpha$.ltime $],|\alpha(0)| \leq \delta$ implies $|\alpha(t)| \leq \epsilon . \mathcal{A}$ is asymptotically stable if it is stable and $\delta$ can be chosen so that, $|\alpha(0)| \leq \delta$ implies $\alpha(t) \rightarrow 0$ as $t \rightarrow \infty$. If the above condition holds for all $\delta$ then $\mathcal{A}$ is globally asymptotically stable.

Uniform stability guarantees that the stability property in question holds for execution fragments and not only for executions. $\mathcal{A}$ is uniformly stable if for every $\epsilon>0$ there exists a constant $\delta>0$, such that for any execution fragment $\alpha, \mid \alpha$.f state $\mid \leq \delta$ implies $\mid \alpha . l$ state $\mid \leq \epsilon$. An SHA $\mathcal{A}$ is said to be uniformly asymptotically stable if it is uniformly stable and there exists a $\delta>0$, such that for every $\epsilon>0$ there exists a $T$, such that for any execution fragment $\alpha$ with $\alpha$. ltime $\geq T, \forall t \geq T, \mid \alpha$.f state $\mid \leq \delta$ implies $|\alpha(t)| \leq \epsilon$. It is said to be globally uniformly asymptotically stable if the above holds for all $\delta$, with $T=T(\delta, \epsilon)$.

It is well known that a hybrid system is stable if all the individual modes of the system are stable and the switching is sufficiently slow, so as to allow the dissipation of the transient effects after each switch. The dwell time [14] and the average dwell time [6] criteria define restricted classes of switching patterns, based on switching speeds, and one can conclude the stability of a system with respect to these restricted classes.

Definition 3. Given a duration of time $\tau_{a}>0, S H A \mathcal{A}$ has Average Dwell Time (ADT) $\tau_{a}$ if there exists a positive constant $N_{0}$, such that for every reachable execution fragment $\alpha, N(\alpha) \leq N_{0}+\alpha$.ltime $/ \tau_{a}$, where $N(\alpha)$ is the number of mode switches in $\alpha$. The number of extra switches of $\alpha$ with respect to $\tau_{a}$ is $S_{\tau_{a}}(\alpha) \triangleq N(\alpha)-\alpha$. ltime $/ \tau_{a}$. 
Theorem 1 from [6], adapted to SHA, gives a sufficient condition for stability based on ADT. Roughly, it states that a hybrid system is stable if the modes are individually stable and the switches do not occur too frequently on the average. See Section 3.2 of [9] for a proof ${ }^{4}$.

Theorem 1. If there exist positive definite, radially unbounded, and continuously differentiable functions $\mathcal{V}_{i}: R^{n} \rightarrow R^{n}$, for each $i \in \mathcal{P}$, and positive numbers $\lambda_{0}$ and $\mu$ such that:

$$
\begin{aligned}
& \frac{\partial \mathcal{V}_{i}}{\partial \mathbf{x}_{c}} f_{i}\left(\mathbf{x}_{c}\right) \leq-\lambda_{0} \mathcal{V}_{i}\left(\mathbf{x}_{c}\right), \quad \forall \mathbf{x}_{c}, \quad \forall i \in \mathcal{P}, \text { and } \\
& \mathcal{V}_{i}\left(\mathbf{x}_{c}^{\prime}\right) \leq \mu \mathcal{V}_{j}\left(\mathbf{x}_{c}\right), \quad \forall \mathbf{x} \stackrel{a}{\rightarrow} \mathcal{A} \mathbf{x}^{\prime}, \text { where } i=\mathbf{x}^{\prime}\lceil\text { mode and } j=\mathbf{x}\lceil\text { mode. }
\end{aligned}
$$

Then, $\mathcal{A}$ is globally uniformly asymptotically stable if it has an $A D T \tau_{a}>\frac{\log \mu}{\lambda_{0}}$.

This stability condition effectively allows us to decouple the construction of Lyapunov functions - the $\mathcal{V}_{i}$ 's for each $i \in \mathcal{P}$, which we assume are known from available methods of system theory - from the problem of checking that the automaton has a certain ADT, which we discuss in the rest of the paper.

\section{ADT: Optimization and Equivalence}

From Definition 3 it follows that a given $\tau_{a}>0$ is not an ADT of a given SHA $\mathcal{A}$ if and only if, for every $N_{0}>0$ there exists a reachable execution fragment $\alpha$ of $\mathcal{A}$ such that $S_{\tau_{a}}(\alpha)>N_{0}$. Thus, if we solve the following optimization problem:

$$
\operatorname{OPT}\left(\tau_{\mathrm{a}}\right): \quad \alpha^{*} \in \arg \max S_{\tau_{a}}(\alpha)
$$

over all the execution fragments of $\mathcal{A}$, and the optimal value $S_{\tau_{a}}\left(\alpha^{*}\right)$ turns out to be bounded, then we can conclude that $\mathcal{A}$ has $\mathrm{ADT} \tau_{a}$, with $S_{\tau_{a}}\left(\alpha^{*}\right)$ as the corresponding value of the constant $N_{0}$. Otherwise, if $S_{\tau_{a}}\left(\alpha^{*}\right)$ is unbounded and $\alpha^{*}$ is reachable then we can conclude that $\tau_{a}$ is not an ADT for $\mathcal{A}$. However, the optimization problem $\mathrm{OPT}\left(\tau_{a}\right)$ may not be solvable using standard mathematical programming tools because, among other things, the executions of $\mathcal{A}$ may not have any finite descriptions. In Sections 4 and 5 we study particular classes of SHA for which OPT $\left(\tau_{a}\right)$ can be solved, but prior to that we define what it means for two SHA to be ADT-equivalent and we develop a simulation relation-based technique for proving this relationship.

Definition 4. Consider $S H A \mathcal{A}$ and $\mathcal{B} . \mathcal{B}$ is faster than $\mathcal{A}$, written as $\mathcal{B} \geq_{A D T}$ $\mathcal{A}$, if for all $\tau_{a}>0, \tau_{a}$ is an $A D T$ for $\mathcal{B}$ implies that $\tau_{a}$ is an $A D T$ for $\mathcal{A}$. If $\mathcal{B} \geq_{A D T} \mathcal{A}$ and $\mathcal{A} \geq_{A D T} \mathcal{B}$, then $\mathcal{A}$ and $\mathcal{B}$ are $\mathrm{ADT}$-equivalent, and this is written as $\mathcal{A}={ }_{A D T} \mathcal{B}$.

\footnotetext{
${ }^{4}$ In [6] and [9] this theorem is presented for the case when discrete transitions do not change the valuation of the continuous variables, but the same proof establishes the result stated here.
} 
Definition 5. A switching simulation relation from $\mathcal{A}$ to $\mathcal{B}$ is a relation $\mathcal{R} \subset$ $Q_{\mathcal{A}} \times Q_{\mathcal{B}}$ satisfying the following conditions:

1. If $\mathbf{x} \in \Theta_{\mathcal{A}}$ then there exists a $\mathbf{y} \in \Theta_{\mathcal{B}}$ such that $\mathbf{x} \mathcal{R} \mathbf{y}$.

2. If $\mathbf{x} \mathcal{R} \mathbf{y}$ and $\alpha$ is an execution fragment of $\mathcal{A}$ consisting of a single action surrounded by two point trajectories with $\alpha$.f state $=\mathbf{x}$, then $\mathcal{B}$ has a closed execution fragment $\beta$, such that $\beta$.f state $=\mathbf{y}, N(\beta) \geq N(\alpha)$, $\beta$.ltime $=0$, and $\alpha . l$ state $\mathcal{R}$ B.lstate. Here $N(\beta)$ is the number of mode switches in $\beta$.

3. If $\mathbf{x} \mathcal{R} \mathbf{y}$ and $\alpha$ is an execution fragment of $\mathcal{A}$ consisting of a single closed trajectory with $\alpha$.f state $=\mathbf{x}$, then $\mathcal{B}$ has a closed execution fragment $\beta$, such that $\beta$.f state $=\mathbf{y}$, $\beta$.ltime $\leq \alpha$.ltime, and $\alpha$.lstate $\mathcal{R}$ B.lstate.

Lemma 1. Let $\mathcal{R}$ be a switching simulation relation from $S H A \mathcal{A}$ to $\mathcal{B}$. Then, for all $\tau_{a}>0$ and for every reachable execution fragment $\alpha$ of $\mathcal{A}$, there exists a reachable execution fragment $\beta$ of $\mathcal{B}$, such that $S_{\tau_{a}}(\beta) \geq S_{\tau_{a}}(\alpha)$.

Proof: We fix $\tau_{a}$ and $\alpha$. Since $\alpha$ is reachable, there exists $\alpha^{\prime}$ such that $\alpha^{\prime} \frown \alpha$ is an execution of $\mathcal{A}$. Let $\alpha=\tau_{0} a_{1} \tau_{1} a_{2} \tau_{2} \ldots$ and let $\alpha$.f state $=\mathbf{x}$. We construct execution $\beta^{\prime} \frown \beta$ of $\mathcal{B}$ such that $\beta$, a reachable execution fragment, has more extra switches than $\alpha$. Since $\alpha^{\prime}$.f state $\in \Theta$, we can find $\beta^{\prime}$ to be such that $\alpha^{\prime}$.f state $\mathcal{R} \beta^{\prime}$.f state and $\alpha^{\prime}$.lstate $\mathcal{R} \beta^{\prime}$.lstate. Therefore, we have $\mathbf{x} \mathcal{R} \beta$.f state. Now, We consider cases for $\alpha$ to show that $S_{\tau_{a}}(\beta) \geq S_{\tau_{a}}(\alpha)$ :

1. $\alpha$ is an infinite sequence.

We can write $\alpha$ as an infinite concatenation $\alpha_{0} \frown \alpha_{1} \frown \alpha_{2} \ldots$, in which the execution fragments $\alpha_{i}$ with $i$ even consist of a trajectory only, and the execution fragments $\alpha_{i}$ with $i$ odd consist of a single discrete transition surrounded by two point trajectories.

We define inductively a sequence $\beta_{0} \beta_{1} \beta_{2}$. of closed execution fragments of $\mathcal{B}$ such that for all $i, \beta_{i}$.lstate $=\beta_{i+1}$.f state, $\alpha_{i}$.lstate $\mathcal{R} \beta_{i}$.lstate, and $S_{\tau_{a}}(\beta) \geq S_{\tau_{a}}(\alpha)$. We use Property 3 of the definition of switching simulation for the construction of the $\beta_{i}$ 's with $i$ even. This gives us $\beta_{i}$.ltime $\leq \alpha_{i}$.ltime for every even $i$. We use Property 2 of the definition of switching simulation for the construction of the $\beta_{i}$ 's with $i$ odd. This gives us $\beta_{i}$.ltime $=\alpha_{i}$.ltime and $N\left(\beta_{i}\right) \geq N\left(\alpha_{i}\right)$ for every odd $i$. Let $\beta=\beta_{0} \frown \beta_{1} \frown \beta_{2} \ldots$, which is clearly an execution fragment for $\mathcal{B}$. Since $\beta$.ltime $\leq \alpha$.ltime and $N(\beta) \geq N(\alpha)$, the required property follows.

2. $\alpha$ is a finite sequence ending with a closed trajectory.

Similar to first case.

3. $\alpha$ is a finite sequence ending with an open trajectory.

Similar to first case, except that the final open trajectory of $\beta$ is constructed using Lemma 4.22 of [8].

Theorem 2 states that the existence of a switching simulation relation from $\mathcal{A}$ to $\mathcal{B}$ is sufficient to establish that $\mathcal{B}$ is faster than $\mathcal{A}$.

Theorem 2. If $\mathcal{R}$ is a switching simulation relation from $\mathcal{A}$ to $\mathcal{B}$, then $\mathcal{B} \geq_{A D T} \mathcal{A}$. 
Proof: We fix a $\tau_{a}$. Given $N_{0}$ such that for every reachable execution fragment $\beta$ of $\mathcal{B}, S_{\tau_{a}}(\beta) \leq N_{0}$, we show that for every reachable execution fragment $\alpha$ of $\mathcal{A}, S_{\tau_{a}}(\alpha) \leq N_{0}$. We fix $\alpha$. From Lemma 1 we know that there exists a $\beta$ such that $S_{\tau_{a}}(\beta) \geq S_{\tau_{a}}(\alpha)$. Since $N_{0} \geq S_{\tau_{a}}(\beta)$ the result follows.

Corollary 1. Let $\mathcal{R}_{1}$ be a switching simulation from $\mathcal{A}$ to $\mathcal{B}$ and $\mathcal{R}_{2}$ be a switching simulation from $\mathcal{B}$ to $\mathcal{A}$. Then, $\mathcal{A}={ }_{A D T} \mathcal{B}$.

\section{Graph SHA and negative cost cycles}

We introduce a special class of SHA, called Graph SHA for which OPT $\left(\tau_{a}\right)$ (see Section 3) can be solved using classical graph algorithms. We show how Graph SHA and switching simulations can be used to prove ADT properties of a linear, scale-independent hysteresis switch.

Graph SHA model. Consider a graph $G$ defined by: a set of vertices $\mathcal{V}$, a set of directed edges $\mathcal{E} \subseteq \mathcal{V} \times \mathcal{V}$, a cost function $w: \mathcal{E} \rightarrow R_{\geq 0}$ for the edges, and a special start edge $e_{0} \in \mathcal{E}$. The cost of a path in $G$ is the sum of the costs of the edges in the path. Given $G=\left(\mathcal{V}, \mathcal{E}, w, e_{0}\right)$, the corresponding Graph SHA $\operatorname{Aut}(G)$ is specified by the code in Figure 1 . The source and the target vertices of an edge $e$ are denoted by $e[1]$ and $e[2]$, respectively. The discrete transitions are written using the standard precondition-effect style. Each trajdef $d$ defines a set of trajectories $\mathcal{T}_{d}$ in terms of the invariant $i n v(d)$, the stopping condition stop $(d)$, and the state model written as an evolve clause evolve $(d)$. A trajectory $\tau$ is in $\mathcal{T}_{d}$, if an only if (1) $\tau$ satisfies evolve $(d),(2) \forall t \in \tau$.dom, $\tau(t) \in \operatorname{inv}(d)$, and (3) $\exists t \in \tau$.dom, $\tau(t) \in \operatorname{stop}(d) \rightarrow t=\tau$.ltime. The set of trajectories of $\operatorname{Aut}(G)$ is the union of the sets of trajectories defined by each trajdef.

Intuitively, the state of $A u t(G)$ captures the motion of a particle moving with unit speed along the edges of the graph $G$. The position of the particle is given by the mode, which is the edge it resides on, and the value of $x$, which is its distance from the source vertex of the edge $G$. Thus, a switch from mode $e$ to mode $e^{\prime}$ of $\operatorname{Aut}(G)$ corresponds to the particle arriving at vertex $e[2]$ via edge $e$, and departing on edge $e^{\prime}$. Within edge $e$ the particle moves at unit speed from $e[1]$, where $x=0$ to $e[2]$, where $x=w(e)$. The next theorem implies that to search

\begin{tabular}{ll} 
Variables: & Precondition \\
mode $\in \mathcal{E} \subset \mathcal{V} \times \mathcal{V}$, initialy $e_{0}$ & mode $=e \wedge e[2]=e^{\prime}[1] \wedge x=w(e)$ \\
$x \in R$, initially 0 & Effect \\
& mode $\leftarrow e^{\prime}, x \leftarrow 0$ \\
Actions: & \\
switch $\left(e, e^{\prime}\right), e, e^{\prime} \in \mathcal{E}$ & Trajectories: \\
& Trajdef edge $($ mode $)$ \\
Transitions: & Evolve $d(x)=1$ \\
switch $\left(e, e^{\prime}\right)$ & Invariant $x \leq w($ mode $)$ \\
& Stop when $x=w($ mode $)$ \\
\hline
\end{tabular}

Fig. 1. Graph SHA $A u t(G)$, where $G=\left(\mathcal{V}, \mathcal{E} \subseteq \mathcal{V} \times \mathcal{V}, w: \mathcal{E} \rightarrow R_{\geq 0}\right.$, and $\left.e_{0}\right)$ 
for an execution of $\operatorname{Aut}(G)$ that violates a ADT property $\tau_{a}$, it is necessary and sufficient to search over the space of the cycles of $G$.

Theorem 3. Consider $\tau_{a}>0$ and a graph SHA Aut $(G)$. $\mathcal{A}$ has average dwell time $\tau_{a}$ if and only if for all $m>1$, the cost of any cycle of $G$ with $m$ segments is at least $m \tau_{a}$.

Proof: First we show that, if there exists a cycle of $G, \beta=e_{0} e_{1} \ldots e_{m}$, such that the cost $\sum_{i=1}^{m} w\left(e_{i}\right)<m \tau_{a}$, then $\tau_{a}$ is not an ADT for $\operatorname{Aut}(G)$. Since $\beta$ is a cycle with $e_{0}[1]=e_{m}[2]$, we know that $\beta$ corresponds to an execution fragment $\alpha$ of $\operatorname{Aut}(G)$, with $\alpha$.fstate $\lceil$ mode $=\alpha$.lstate $\lceil$ mode and $\alpha$.f state $\left\lceil x=\alpha\right.$.lstate $\left\lceil x=0\right.$. Clearly, $S_{\tau_{a}}(\alpha)=\alpha$. length $-\alpha$. ltime $/ \tau_{a}=$ $m-\frac{1}{\tau_{a}} \sum_{i=1}^{n} \tau_{i}$. ltime, this equals $\frac{1}{\tau_{a}}\left(m \tau_{a}-\sum_{i=1}^{m} w\left(e_{i}\right)\right.$, which is positive. Therefore we can construct an execution $\gamma$ of $\operatorname{Aut}(G)$ by concatenating $\beta \frown \beta \neg \beta \ldots$, $k$ times. So, $S_{\tau_{a}}(\gamma)=k S_{\tau_{a}}(\alpha)$ which can be made arbitrarily large by increasing $k$. So, $\tau_{a}$ is not an ADT for $\operatorname{Aut}(G)$.

We show that if $\tau_{a}$ is not an ADT for $\operatorname{Aut}(G)$, then there exists a cycle in $G$ such that, if the length of the cycle is $m$, then its cost $\sum_{i=1}^{m} w_{i}$ is less than $m \tau_{a}$. We choose $c_{0}$ to be larger than the number of edges $|\mathcal{E}|$ in $\bar{G}$. Of all the execution fragments that have more than $c_{0}$ extra switches with respect to $\tau_{a}$, let $\alpha$ be the shortest. Let $\beta$ be the path of $G$ corresponding $\alpha$. Suppose the length of $\beta$ is $l$. Since $l-\frac{1}{\tau_{a}} \sum_{i=1}^{l} w\left(e_{i}\right)>c_{0}$ and $c_{0}>|\mathcal{E}|$, some of the vertices must be repeated in $\beta$. That is, $\beta$ must contain a cycle. Suppose $\beta=\beta_{p} \cdot \gamma \cdot \beta_{s}$, where $\gamma$ is cycle. Let $l_{1}, l_{2}, l_{3}$ be the lengths of $\beta_{p}, \gamma$, and $\beta_{s}$, respectively. For the sake of contradiction we assume that, the cost of the cycle $\gamma, \sum_{i=1}^{l_{2}} w\left(e_{i}\right) \geq l_{2} \tau_{a}$. Therefore,

$$
\begin{aligned}
& l>c_{0}+\frac{1}{\tau_{a}} \sum_{\beta} w_{i} \\
& l_{1}+l_{2}+l_{3}>c_{0}+\frac{1}{\tau_{a}} \sum_{i=1}^{l_{1}} w_{i}+\frac{1}{\tau_{a}} \sum_{i=1}^{l_{2}} w_{i}+\frac{1}{\tau_{a}} \sum_{i=1}^{l_{3}} w_{i} \\
& l_{1}+l_{3}+\frac{1}{\tau_{a}}\left(m \tau_{a}-\sum_{i=1}^{l_{2}} w_{i}\right)>c_{0}+\sum_{\beta_{p}, \beta_{s}} w_{i} \\
& l_{1}+l_{3}>c_{0}+\frac{1}{\tau_{a}}\left[\sum_{i=1}^{l_{1}} w_{i}+\sum_{i=1}^{l_{3}} w_{i}\right]
\end{aligned}
$$

From Equation (1), $S_{\tau_{a}}\left(\beta_{p} \frown \beta_{s}\right)>c_{0}$, and we already know that $\beta_{p} \frown \beta_{s}$ is shorter than $\beta$. Therefore, there exists an fragment execution $\alpha^{\prime}$ of $\operatorname{Aut}(G)$ that has $S_{\tau_{a}}\left(\alpha^{\prime}\right)>c_{0}$ and $\alpha^{\prime}$.length $<\alpha$.length. This contradicts our assumption that $\alpha$ is the shortest fragment execution with more than $c_{0}$ extra switches.

Thus, the problem of solving $\mathrm{OPT}\left(\tau_{a}\right)$ for $\operatorname{Aut}(G)$ reduces to the problem of checking whether $G$ contains a cycle of length $m$, for some $m>1$, with cost less than $m \tau_{a}$. We can solve this problem as follows: we scale the weight of every edge $e$ of $G$ to be $\frac{w(e)}{\tau_{a}}-1$, and check for the existence of a negative cost cycle in the new graph. Existence of a negative cost cycle in any graph $G=(\mathcal{V}, \mathcal{E})$ can be detected efficiently using the Bellman-Ford algorithm [3] in $O(\mathcal{V E})$ time. 
Now we specify a Graph automaton $\mathcal{B}$, in terms of the parameters of $\mathcal{A}$, that captures the switching behavior of $\mathcal{A}$. Consider a graph $G=\left(\mathcal{V}, \mathcal{E}, w, e_{0}\right)$, where:

1. $\mathcal{V} \subset\{1,(1+h)\}^{n}$, such that for any $v \in V$, all the $n$-components are not equal. We denote the $i^{\text {th }}$ component of $v \in V$, by $v[i]$.

2. An edge $(u, v) \in \mathcal{E}$ if and only if, one of the following conditions hold:

(a) There exists $j \in\{1, \ldots, n\}$, such that, $u[j] \neq v[j]$ and for all $i \in\{1, \ldots, n\}$,

$i \neq j, u[i]=v[i]$. The cost of the edge $w(u, v) \triangleq \frac{1}{c_{j}} \ln (1+h)$ and we define $\zeta(u, v) \triangleq j$.

(b) There exists $j \in\{1, \ldots, n\}$ such that $u[j]=1, v[j]=(1+h)$ and for all $i \in$ $\{1, \ldots, n\}, i \neq j$ implies $u[i]=(1+h)$ and $v[i]=1$. The cost of the edge $w(u, v) \triangleq$ $\frac{2}{c_{j}} \ln (1+h)$ and we define $\zeta(u, v) \triangleq j$.

3. $e_{0} \in \mathcal{E}$, such that $e_{0}[1]\left[i_{0}\right]=(1+h)$ and for all $i \neq p_{0}, e_{0}[1][i]=1$.

As an example, the graph for $n=3$ is shown in Figure 3 . Let $\mathcal{B}$ be the Graph SHA $A u t(G)$. Each edge of $G$ corresponds to a mode of $\mathcal{A}$. In fact, mode of $\mathcal{A}$ equals $\zeta(e)$, where $e$ is the edge corresponding to the mode of $\mathcal{B}$. We define a

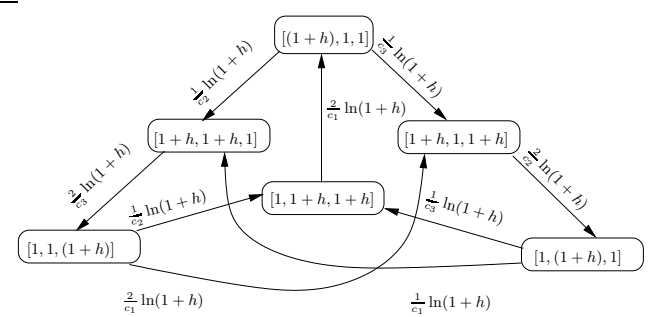

Fig. 3. Graph $G$ with 3 modes. Here $h$ and $c_{i}$ 's are the parameters from the hysteresis switch automaton $\mathcal{A}$.

relation $\mathcal{R}$ on the state spaces on $\mathcal{A}$ and $\mathcal{B}$. Each vertex of $G$ is an $n$-tuple; the $i^{\text {th }}$ component of the source vertex of $e$ is denoted by $e[1][i]$.

Definition 7. For any $\mathbf{x} \in Q_{\mathcal{A}}$ and $\mathbf{y} \in Q_{\mathcal{B}}, \mathbf{x} \mathcal{R} \mathbf{y}$ if and only if:

1. $\zeta(\mathbf{y}\lceil$ mode $)=\mathbf{x}\lceil$ mode

2. For all $j \in\{1, \ldots, n\}$, if $j=\zeta\left(\mathbf{y}\lceil\right.$ mode $)$ then (a) $\frac{\mathbf{x}\left\lceil\mu_{j}\right.}{\mathbf{x}\left\lceil\mu_{\min }\right.}=e^{c_{j}(\mathbf{y}\lceil x)}$ else (b) $\frac{\mathbf{x}\left\lceil\mu_{j}\right.}{\mathbf{x}\left\lceil\mu_{\min }\right.}=\left(\mathbf{y}\lceil\operatorname{mode})[1][j]\right.$ and $\frac{\mathbf{x}\left\lceil\mu_{j}\right.}{\mathbf{x}\left\lceil\mu_{\min }\right.}=(\mathbf{y}\lceil\operatorname{mode})[2][j]$.

Part 1 of Definition 7 states that if $\mathcal{A}$ is in mode $j$ and $\mathcal{B}$ is in mode $e$, then $\zeta(e)=j$. Part 2 states that for all $j \neq \zeta(e)$, the $j^{\text {th }}$ component of $e[1]$ and $e[2]$ are the same, and are equal to $\mu_{j} / \mu_{\min }$, and for $j=\zeta(e), \mu_{j}=\mu_{\min } e^{c_{j} x}$.

Lemma 2. $\mathcal{R}$ is a switching simulation relation from $\mathcal{A}$ to $\mathcal{B}$.

Proof: We show that $\mathcal{R}$ is a switching simulation relation from $\mathcal{A}$ to $\mathcal{B}$. $\mathcal{A}$ has a unique start state and it is easy to see that it is related to all the start states 
of $\mathcal{B}$. Next we show by cases that given any state $\mathbf{x} \in Q_{\mathcal{A}}, \mathbf{y} \in Q_{\mathcal{B}}, \mathbf{x} \mathcal{R} \mathbf{y}$, and an execution fragment $\alpha$ of $\mathcal{A}$ starting from $\mathbf{x}$ and consisting of either a single action or a single trajectory, there exists a corresponding execution fragment $\beta$ of $\mathcal{B}$, starting from $\mathbf{y}$ that satisfies the conditions required for $\mathcal{R}$ to be a switching simulation relation.

1. $\alpha$ is a $\left(\mathbf{x}, \operatorname{switch}(i, j), \mathbf{x}^{\prime}\right)$ transition of $\mathcal{A}$ and $i$ is not the unique minimum at $\mathbf{x}$ and $j$ is not unique minimum at $\mathbf{x}^{\prime}$.

We choose $\beta$ to be $\left(\mathbf{y}, \operatorname{switch}\left(e, e^{\prime}\right), \mathbf{y}^{\prime}\right)$ action of $\mathcal{B}$, where $e$ and $e^{\prime}$ are determined by the following rules:

$$
\begin{gathered}
e[1][i]=1, e[2][i]=1+h, \forall k, k \neq i, e[1][k]=e[2][k]=\frac{\mathbf{x}\left\lceil\mu_{k}\right.}{\mathbf{x}\left\lceil\mu_{\min }\right.} \\
e^{\prime}[1][j]=1, e^{\prime}[2][j]=1+h, \forall k, k \neq j, e^{\prime}[1][k]=e^{\prime}[2][k]=\frac{\mathbf{x}^{\prime}\left\lceil\mu_{k}\right.}{\mathbf{x}^{\prime}\left\lceil\mu_{\min }\right.}
\end{gathered}
$$

We have to show that switch $\left(e, e^{\prime}\right)$ is enabled at $\mathbf{y}$; this involves showing that the three conjuncts in the precondition of the switch action of $\mathcal{B}$ are satisfied at $\mathbf{y}$. First of all, since $\mathbf{x} \mathcal{R} \mathbf{y}$ we know that $\zeta(\mathbf{y}\lceil$ mode $)=\mathbf{x}\lceil$ mode $=i$. Further, $i$ is not a unique minimum at $\mathbf{x}$, so from the definition of the edges of $G$ it follows that:

$$
\begin{gathered}
(\mathbf{y}\lceil\operatorname{mode})[1][i]=1,(\mathbf{y}\lceil\operatorname{mode})[2][i]=i+h, \\
\forall k, k \neq i,\left(\mathbf{y}\lceil\text { mode })[1][k]=\left(\mathbf{y}\lceil\operatorname{mode})[2][k]=\frac{\mathbf{x}\left\lceil\mu_{k}\right.}{\mathbf{x}\left\lceil\mu_{\min }\right.}\right.\right.
\end{gathered}
$$

Comparing Equations (4) and (10) we conclude that $\mathbf{y}\lceil\operatorname{mode}=e$.

Secondly, using the definitions of $e, e^{\prime}$ and $\mathcal{R}$ it follows that:

$$
e[2][i]=1+h=\frac{\mathbf{x}\left\lceil\mu_{i}\right.}{\mathbf{x}\left\lceil\mu_{\min }\right.}=\frac{\mathbf{x}^{\prime}\left\lceil\mu_{i}\right.}{\mathbf{x}^{\prime}\left\lceil\mu_{\min }\right.}=e^{\prime}[1][i]
$$

The second equality holds because $\operatorname{switch}(i, j)$ is enabled at $\mathbf{x}$. The third equality follows from the fact that the $\operatorname{switch}(i, j)$ transition of $\mathcal{A}$ does not alter the value of the $\mu_{k}$ 's. Likewise, we have:

$$
\begin{array}{r}
e[2][j]=\frac{\mathbf{x}\left\lceil\mu_{j}\right.}{\mathbf{x}\left\lceil\mu_{\min }\right.}=\frac{\mathbf{x}^{\prime}\left\lceil\mu_{j}\right.}{\mathbf{x}^{\prime}\left\lceil\mu_{\min }\right.}=1=e^{\prime}[1][j] \\
\forall k, k \neq j, k \neq i, e[2][k]=\frac{\mathbf{x}\left\lceil\mu_{k}\right.}{\mathbf{x}\left\lceil\mu_{\min }\right.}=\frac{\mathbf{x}^{\prime}\left\lceil\mu_{k}\right.}{\mathbf{x}^{\prime}\left\lceil\mu_{\min }\right.}=e^{\prime}[1][k]
\end{array}
$$

Combining Equations (5),(5) and (7) it follows that $e[2]=e^{\prime}[1]$.

Finally, from the switching simulation relation $\mathcal{R}$, we know that $\mathbf{y}\lceil x=$ $\frac{1}{c_{i}} \ln \frac{\mathbf{x}\left\lceil\mu_{i}\right.}{\mathbf{x}\left\lceil\mu_{\min }\right.}=\frac{1}{c_{i}} \ln (1+h)$. And since $\zeta(e)=i$ and $i$ is not the unique minimum at $\mathbf{x}$, from the definition of the edge costs of $G$ it follows that $\mathbf{y}\left\lceil x=w(e)\right.$. Thus, we have shown that $\operatorname{switch}\left(e, e^{\prime}\right)$ is indeed enabled at $\mathbf{y}$. Next, we have to show that $\mathbf{x}^{\prime} \mathcal{R} \mathbf{y}^{\prime}$. First of all, $\mathbf{x}^{\prime}\left\lceil\operatorname{mode}=j\right.$ and $\mathbf{y}^{\prime}\lceil$ mode $=e^{\prime}$ from the effect parts of the $\operatorname{switch}(i, j)$ and $\operatorname{switch}\left(e, e^{\prime}\right)$ actions, respectively. Also, $\zeta\left(e^{\prime}\right)=j$ from Equation (10). It follows that $\mathbf{x}^{\prime}\lceil$ mode $=$ $\zeta\left(\mathbf{y}^{\prime}\lceil\right.$ mode $)$. Secondly, $\frac{\mathbf{x}^{\prime}\left\lceil\mu_{j}\right.}{\mathbf{x}^{\prime}\left\lceil\mu_{\text {min }}\right.}=\frac{\mathbf{x}\left\lceil\mu_{j}\right.}{\mathbf{x}\left\lceil\mu_{\min }\right.}=1$, from the precondition of 
$\operatorname{switch}(i, j)$. Since $\mathbf{y}^{\prime}\left\lceil x=0\right.$ it follows that $\frac{\mathbf{x}\left\lceil\mu_{j}\right.}{\mathbf{x}\left\lceil\mu_{m i n}\right.}=e^{c_{j} \mathbf{y}^{\prime}\lceil x}$. Finally, for all $k \neq j$, again from Equation (10) it follows that $\frac{\mathbf{x}^{\prime}\left\lceil\mu_{k}\right.}{\mathbf{x}^{\prime} \mid \mu_{\min }}=e^{\prime}[1][k]=e^{\prime}[2][k]$.

2. $\alpha$ is a $\left(\mathbf{x}, \operatorname{switch}(i, j), \mathbf{x}^{\prime}\right)$ transition of $\mathcal{A}$ and $i$ is the unique minimum at $\mathbf{x}$ and $j$ is not unique minimum at $\mathbf{x}^{\prime}$.

We choose $\beta$ to be $\left(\mathbf{y}, \operatorname{switch}\left(e, e^{\prime}\right), \mathbf{y}^{\prime}\right)$ action of $\mathcal{B}$, where $e$ and $e^{\prime}$ are determined by the following rules:

$$
\begin{gathered}
e[1][i]=1, e[2][i]=1+h, \forall k, k \neq i, e[1][k]=(1+h), e[2][k]=1 \\
e^{\prime}[1][j]=1, e^{\prime}[2][j]=1+h, \forall k, k \neq j, e^{\prime}[1][k]=e^{\prime}[2][k]=\frac{\mathbf{x}^{\prime}\left\lceil\mu_{k}\right.}{\mathbf{x}^{\prime}\left\lceil\mu_{\min }\right.}
\end{gathered}
$$

The rest of the proof is similar to that of case 1 .

3. $\alpha$ is a $\left(\mathbf{x}, \operatorname{switch}(i, j), \mathbf{x}^{\prime}\right)$ transition of $\mathcal{A}$ and $i$ is not the unique minimum at $\mathbf{x}$ and $j$ is the unique minimum at $\mathbf{x}^{\prime}$.

We choose $\beta$ to be $\left(\mathbf{y}\right.$, switch $\left.\left(e, e^{\prime}\right), \mathbf{y}^{\prime}\right)$ action of $\mathcal{B}$, where $e$ and $e^{\prime}$ are determined by the following rules:

$$
\begin{gathered}
e[1][i]=1, e[2][i]=1+h, \forall k, k \neq i, e[1][k]=e[2][k]=\frac{\mathbf{x}\left\lceil\mu_{k}\right.}{\mathbf{x}\left\lceil\mu_{m i n}\right.} \\
e^{\prime}[1][j]=1, e^{\prime}[2][j]=1+h, \forall k, k \neq j, e^{\prime}[1][k]=(1+h), e^{\prime}[2][k]=1
\end{gathered}
$$

The rest of the proof is similar to that of case 1 .

4. $\alpha$ is a closed trajectory $\tau$ of $\mathcal{A}$ with $(\tau \downarrow$ mode $)(0)=i$ for some $i \in \mathcal{P}$, such that $i$ is not unique minimum at $\tau$.f state.

We choose $\beta$ to be the trajectory $\tau^{\prime}$ of $\mathcal{B}$ with $\tau^{\prime}$.dom $=\tau$.dom determined by the following rules. Let $\mathbf{x}=\tau$.f state, $\mathbf{x}^{\prime}=\tau$.lstate, $\mathbf{y}=\tau^{\prime}$. fstate and $\mathbf{y}^{\prime}=\tau^{\prime}$. lstate.

$$
\begin{gathered}
(\mathbf{y}\lceil\text { mode })[1][i]=1,(\mathbf{y}\lceil\operatorname{mode})[2][i]=1+h, \\
\forall k, k \neq i,\left(\mathbf{y}\lceil\text { mode })[1][k]=\left(\mathbf{y}\lceil\operatorname{mode})[1][k]=\frac{\mathbf{x}\left\lceil\mu_{i}\right.}{\mathbf{x}\left\lceil\mu_{\min }\right.},\right.\right. \\
\forall t \in \tau^{\prime} . \operatorname{dom},\left(\tau^{\prime} \downarrow x\right)(t)=\frac{1}{c_{i}} \ln \frac{\mathbf{x}\left\lceil\mu_{i}\right.}{\mathbf{x}\left\lceil\mu_{\min }\right.}+t
\end{gathered}
$$

We first show that $\tau^{\prime}$ is a valid trajectory of $\mathcal{B}$. First of all, it is easy to check that $\tau^{\prime}$ satisfies the constant differential equation $d(x)=1$ and that the mode of $\mathcal{B}$ remains constant. Next, we show that $\tau^{\prime}$ satisfies the stopping condition " $x=w($ mode $)$ ". Suppose there exists $t \in \tau^{\prime}$.dom such that $(\tau\lceil$ $x)(t)=w(\mathbf{x}\lceil$ mode $)$, then $t=w(\mathbf{x}\lceil$ mode $)-(\mathbf{y}\lceil x)$. Then,

$$
\begin{aligned}
\mathbf{x}^{\prime}\left\lceil\mu_{i}\right. & =\mathbf{x}\left\lceil\mu_{i} e^{c_{i} t}\right. \\
\frac{1}{c_{i}} \ln \frac{\mathbf{x}^{\prime}\left\lceil\mu_{i}\right.}{\mathbf{x}\left\lceil\mu_{i}\right.} & =w(\mathbf{x}\lceil\text { mode })-(\mathbf{y}\lceil x) \\
& =\frac{1}{c_{i}} \ln (1+h)-(\mathbf{y}\lceil x) \quad[\text { by replacing } w(\mathbf{x}\lceil\text { mode })] \\
& =\frac{1}{c_{i}}\left[\ln (1+h)-\ln \frac{\mathbf{x}\left\lceil\mu_{i}\right.}{\mathbf{x}\left\lceil\mu_{\min }\right.}\right] \quad[\text { from }(11)] \\
\mathbf{x}^{\prime}\left\lceil\mu_{i}\right. & =(1+h)\left(\mathbf{x}\left\lceil\mu_{\min }\right)\right. \\
& =(1+h)\left(\mathbf{x}^{\prime}\left\lceil\mu_{\min }\right) \quad\left[i \text { not unique min } \Rightarrow \mu_{\min } \text { constant over } \tau^{\prime} .\right]\right.
\end{aligned}
$$


Last equation implies that $\mathbf{x}^{\prime}$ satisfies the stopping condition for trajdef mode(i) for automaton $\mathcal{A}$. Therefore, $t=\tau$.ltime $=\tau^{\prime}$.ltime. Thus we have shown that $\tau^{\prime}$ is a valid trajectory of automaton $\mathcal{B}$.

We show that $\mathbf{x}^{\prime} \mathcal{R} \mathbf{y}^{\prime}$. First, $\mathbf{x}^{\prime}\left\lceil\operatorname{mode}=\zeta\left(\mathbf{y}^{\prime}\lceil\operatorname{mode})\right.\right.$ because $\mathbf{x}^{\prime} \Gamma$ mode $=\mathbf{x}\left\lceil\right.$ mode $=\zeta\left(\mathbf{y}\lceil\right.$ mode $)=\zeta\left(\mathbf{y}^{\prime}\lceil\right.$ mode $)$. Secondly, for all $k, k \neq i$, $\mathbf{x}\left\lceil\mu_{i}=\mathbf{x}^{\prime}\left\lceil\mu_{i}\right.\right.$ and $\left(\mathbf{y}\lceil\operatorname{mode})[1][k]=\left(\mathbf{y}^{\prime}\lceil\operatorname{mode})[1][k]\right.\right.$. Finally, we show that $\mathbf{x}^{\prime}\left\lceil\mu_{i}=\left(\mathbf{x}^{\prime}\left\lceil\mu_{\min }\right) e^{c_{i}\left(\mathbf{y}^{\prime}\lceil x)\right.}\right.\right.$ by reasoning as follows:

$$
\begin{aligned}
\mathbf{x}^{\prime}\left\lceil\mu_{i}\right. & =\left(\mathbf{x}\left\lceil\mu_{i}\right) e^{c_{i} \tau . l t i m e}\right. \\
& =\left(\mathbf{x}\left\lceil\mu_{\text {min }}\right) e^{c_{i}(\mathbf{y}\lceil x+\tau . \text { ltime })}\right. \\
& =\left(\mathbf{x}^{\prime}\left\lceil\mu_{\min }\right) e^{c_{i}\left(\mathbf{y}\left\lceil x+\tau^{\prime} . \text { ltime }\right)\right.}\right.
\end{aligned}
$$

5. $\alpha$ is a closed trajectory $\tau$ of $\mathcal{A}$ with $(\tau \downarrow$ mode $)(0)=i$ for some $i \in \mathcal{P}$, such that $i$ is the unique minimum at $\tau$. fstate.

We choose $\beta$ to be the trajectory $\tau^{\prime}$ of $\mathcal{B}$ with $\tau^{\prime}$.dom $=\tau$.dom determined by the following rules. Let $\mathbf{x}=\tau$.f state and $\mathbf{y}=\tau^{\prime}$. f state.

$$
\begin{gathered}
(\mathbf{y}\lceil\operatorname{mode})[1][i]=1,(\mathbf{y}\lceil\operatorname{mode})[2][i]=1+h, \\
\forall k, k \neq i,(\mathbf{y}\lceil\operatorname{mode})[1][k]=(1+h),(\mathbf{y}\lceil\operatorname{mode})[2][k]=1 \\
\forall t \in \tau^{\prime} . \operatorname{dom},\left(\tau^{\prime} \downarrow x\right)(t)=\frac{1}{c_{i}} \ln \frac{\mathbf{x}\left\lceil\mu_{i}\right.}{\mathbf{x}\left\lceil\mu_{\min }\right.}+t
\end{gathered}
$$

The rest of the proof for this case is similar to that for case 4 .

Lemma 3. $\mathcal{R}$ is a switching simulation relation from $\mathcal{B}$ to $\mathcal{A}$.

The proof is similar to that of Lemma 2 .

Remark 1. From Corollary 1 it follows that SHA $\mathcal{A}$ and the graph SHA $\mathcal{B}$ are ADT-equivalent. We already know that $\mathrm{ADT}$ properties of $\mathcal{B}$ can be automatically verified using Bellman-Ford algorithm. Therefore, we can also verify ADT of $\mathcal{A}$ automatically. The ADT of a linear hysteresis switch with $\mathcal{P}$ modes can be checked in $O\left(2^{|\mathcal{P}|}\right)$ time.

\section{Initialized SHA and mixed integer linear programming}

Given $\tau_{a}>0$ and an Initialized SHA $\mathcal{A}$, we show that existence of a cycle of $\mathcal{A}$ with extra switches with respect to $\tau_{a}$ is necessary and sufficient to conclude that $\tau_{a}$ is not an ADT for $\mathcal{A}$. We apply this result to rectangular initialized SHA to solve the ADT verification problem using Mixed Integer Linear Programming (MILP).

Initialized SHA model. A SHA $\mathcal{A}$ is initialized if every $a \in M$ is associated with a set $R_{a} \subseteq Q$, such that $\mathbf{x} \stackrel{a}{\rightarrow} \mathbf{x}^{\prime}$ is a mode switching transition if and only if $\mathbf{x} \in G_{a}$ and $\mathbf{x}^{\prime} \in R_{a}$. The set $R_{a}$ is called the initialization predicate of $a$. A SHA is rectangular if the differential equations in the state models have constant 
right hand sides, and the guard and the initialization predicates (restricted to the set of continuous variables) are polyhedra.

Theorem 4 implies that for an initialized SHA $\mathcal{A}$, it is necessary and sufficient to solve OPT $\left(\tau_{a}\right)$ over the space of the cyclic fragments of $\mathcal{A}$ instead of the larger space of all execution fragments.

Theorem 4. Given $\tau_{a}>0$ and initialized SHA $\mathcal{A}, \tau_{a}$ is an $A D T$ for $\mathcal{A}$ if and only if $\mathcal{A}$ does not have any cycles with extra switches with respect to $\tau_{a}$.

Proof: For simplicity we assume that all discrete transitions of the automaton $\mathcal{A}$ are mode switching transitions. Existence of a reachable cycle $\alpha$ with extra switches with respect to $\tau_{a}$ is sufficient to show that $\tau_{a}$ is not an $\mathrm{ADT}$ for $\mathcal{A}$. This is because, by concatenating a sequence of $\alpha$ 's we can construct an execution fragment $\alpha^{\frown} \alpha^{\frown} \alpha \ldots$ with an arbitrarily large number of extra switches.

We prove by contradiction that existence of a cycle with extra switches is necessary for violating the ADT property. we assume that $\tau_{a}>0$ is not an average dwell time for $\mathcal{A}$ and that $\mathcal{A}$ does not have any cycles with extra switches. By the definition of $\mathrm{ADT}$, for any constant $N_{0}$ there exists an execution that has more than $N_{0}$ extra switches with respect to $\tau_{a}$. Let us choose $N_{0}>|\mathcal{P}|^{3}$. Of all the executions that have more than $N_{0}$ extra switches, let $\alpha=\tau_{0} a_{1} \tau_{1} \ldots \tau_{n}$ be a closed execution that has the smallest number of mode switches. From $\alpha$, we construct $\beta=\tau_{0}^{*} a_{1} \tau_{1}^{*} \ldots \tau_{n}^{*}$, using the following two rules:

1. Each $\tau_{i}$ of $\alpha$ is replaced by $\tau_{i}^{*}=\min _{\tau \in \mathcal{T}_{\mathcal{A}}} \tau$.ltime, such that $\tau_{i}^{*}$.fstate $\in R_{a_{i}}$ and $\tau_{i}^{*}$. lstate $\in G_{a_{i+1}}$,

2. If there exists $i, j \in\{1, \ldots, n-1\}$, such that $a_{i}=a_{j}$ and $a_{i+1}=a_{j+1}$, then we make $\tau_{i}^{*}=\tau_{j}^{*}$.

Claim. The sequence $\beta$ is an execution fragment of $\mathcal{A}$ and $S_{\tau_{a}}(\beta)>|\mathcal{P}|^{3}$.

Proof of claim: We prove the first part of the claim by showing that each application of the above rules to an execution fragment of $\mathcal{A}$ results in another execution fragment. Consider Rule 1 and fix $i$. Since $\tau_{i}^{*}$.f state $\in R_{a_{i}}$ and $\tau_{i-1}$.lstate $\in G_{a_{i}}$, $\tau_{i-1}$.lstate $\stackrel{a_{i}}{\rightarrow} \tau_{i}^{*}$.f state. And, since $\tau_{i}^{*}$.lstate $\in G_{a_{i+1}}$ and $\tau_{i+1}$.f state $\in R_{a_{i+1}}$, we know that $\tau_{i}^{*}$.lstate $\stackrel{a_{i+1}}{\longrightarrow} \tau_{i+1}$.f state. Now for Rule 2 , we assume there exists $i$ and $j$ such that the hypothesis of the rule holds and suppose $\tau_{j}^{*}=\tau_{i}^{*}=\tau_{i}$. We know that even if $\tau_{j}^{*} \neq \tau_{j}$, the first states of both are in $R_{a_{j}}$ and the last states are in $G_{a_{j+1}}$. Therefore, $a_{j}$ matches up the states of $\tau_{j-1}$ and $\tau_{j}^{*}$ and likewise $a_{j+1}$ matches that states of $\tau_{j}^{*}$ and $\tau_{j+1}$.

The second part of the claim follows from the fact that each trajectory $\tau_{i}$ is replaced by the shortest trajectory $\tau_{i}^{*}$ from the initialization set of the previous transition $R_{a_{i}}$ to the guard set of the next transition $G_{a_{i+1}}$. That is, for each $i$, $0<i<n, \tau_{i}^{*}$.ltime $\leq \tau_{i}$.ltime and therefore $\beta$.ltime $\leq \alpha$.ltime and $S_{\tau_{a}}(\beta)>$ $N_{0}>|\mathcal{P}|^{3}$.

Since $N(\beta)>|\mathcal{P}|^{3}$, there must be a sequence of 3 modes that appear multiple times in $\beta$. That is, there exists $i, j \in\{1, \ldots, n\}$, and $p, q, r \in \mathcal{P}$, such that 
$\tau_{i}^{*} . f$ state $\left\lceil\operatorname{mode}=\tau_{j}^{*} . f\right.$ state $\left\lceil\right.$ mode $=p, \tau_{i+1}^{*} . f$ state $\left\lceil\right.$ mode $=\tau_{j+1}^{*} . f$ state \lceil mode $=q$, and $\tau_{i+2}^{*} . f$ state $\left\lceil\operatorname{mode}=\tau_{j+2}^{*} . f\right.$ state $\lceil\operatorname{mode}=r$. Then, from Rule 2 we know that $\tau_{i+1}^{*}=\tau_{j+1}^{*}$. In particular, $\tau_{i+1}^{*}$.f state $=\tau_{j+1}^{*} . f$ state, that is, we can write $\beta=\beta_{p} \frown \gamma / \beta_{s}$, where $\gamma$ is a cycle. Then we have the following:

$$
\begin{aligned}
& N\left(\beta_{p}\right)+N(\gamma)+N\left(\beta_{s}\right)>N_{0}+\beta_{p} . \text { ltime } / \tau_{a}+\gamma . \text { ltime } / \tau_{a}+\beta_{s} . l \text { ltime } / \tau_{a} \\
& N\left(\beta_{p}\right)+N\left(\beta_{s}\right)+S_{\tau_{a}}(\gamma)>N_{0}+\beta_{p} . \text { ltime } / \tau_{a}+\beta_{s} . l t i m e / \tau_{a} \\
& N\left(\beta_{p} \frown \beta_{s}\right)>N_{0}+\beta_{p} \frown \beta_{s} . l \text { ltime } / \tau_{a} \quad\left[\beta_{p} . l \text { lstate }=\beta_{s} . \text { fstate }\right]
\end{aligned}
$$

The last step follows from the assumption that $S_{\tau_{a}}(\gamma) \leq 0$. Therefore, we have $S_{\tau_{a}}\left(\beta_{p} \frown \beta_{s}\right)>N_{0}$ which contradicts our assumption that $\beta$ has the smallest number of mode switches among all the executions that have more that $N_{0}$ extra switches with respect to $\tau_{a}$.

The next lemma allows us to limit the search for cycles with extra switches to cycles with at most $|\mathcal{P}|^{3}$ mode switches. It is proved by showing that any cycle with extra switches that has more than $|\mathcal{P}|^{3}$ mode switches, can be decomposed into two smaller cycles, one of which must also have extra switches.

Lemma 4. If initialized SHA $\mathcal{A}$ has a cycle with extra switches, then it has a cycle with extra switches that has fewer than $|\mathcal{P}|^{3}$ mode switches.

MILP formulation of $\operatorname{OPT}\left(\tau_{a}\right)$. Figure 4 shows the specification of a generic Initialized rectangular SHA $\mathcal{A}$. The automaton $\mathcal{A}$ has a single discrete variable called mode which takes values in the index set $\mathcal{P}=\{1, \ldots, N\}$, and a continuous variable vector $\mathbf{x} \in R^{n}$. For any $p, q \in \mathcal{P}$, the action that changes the mode from $p$ to $q$ is called switch $(p, q)$. The guard and the initialization predicates of this action are given by sets of linear inequalities on the continuous variables, represented in the matrix notation by: $G[p, q] \mathbf{x} \leq g[p, q]$ and $R[p, q] \mathbf{x} \leq r[p, q]$, respectively, where $G[p, q]$ and $R[p, q]$ are constant matrices with $n$ columns and $g[p, q], r[p, q]$ are constant vectors. For each mode $p \in P$ of automaton $\mathcal{A}$, the

\begin{tabular}{|lc|}
\hline & \\
Variables: & Precondition \\
mode $\in \mathcal{P}$, initially $p$ & mode $=p \wedge G[p, q] \mathbf{x} \leq g[p, q]$ \\
$\mathbf{x} \in R^{n}$, initially $\mathbf{x}_{0}$ & Effect \\
& mode $\leftarrow q$ \\
Actions & $\mathbf{x} \leftarrow \mathbf{x}^{\prime}$ such that $R[p, q] \mathbf{x}^{\prime} \leq r[p, q]$ \\
$\quad$ switch $(p, q), p, q \in \mathcal{P}$ & Trajectories: \\
Transitions: & Trajdef mode $(p)$ \\
switch $(p, q)$ & Invariant $A[p] \mathbf{x} \leq a[p]$ \\
& Evolve $d(\mathbf{x})=c[p]$ \\
\hline
\end{tabular}

Fig. 4. Generic rectangular initialized SHA with parameters $\mathcal{P}, G, A, R, q, a, r, c$.

invariant is stated in terms of linear inequalities of the continuous variables $A[p] \mathbf{x} \leq a[p]$, where $A[p]$ is a constant matrix with $n$ columns and $a[p]$ is a 
constant vector. The evolve clause is given by a single differential equation $d(\mathbf{x})=$ $c[p]$, where the right hand side $c[p]$ is a constant vector.

We describe a MILP formulation $\operatorname{MOPT}\left(K, \tau_{a}\right)$ for finding a cyclic execution with $K$ mode switches that maximizes the number of extra switches with respect to $\tau_{a}$. If the optimal value is positive, then the optimal solution represents a cycle with extra switches with respect to $\tau_{a}$, and we conclude that $\tau_{a}$ is not an ADT for $\mathcal{A}$. On the other hand, if the optimal value is not positive, then we conclude that there are no cycles with extra switches of length $K$. To verify ADT of $\mathcal{A}$, we solve a sequence of $\operatorname{MOPT}\left(K, \tau_{a}\right)$ 's with $K=2, \ldots,|\mathcal{P}|^{3}$. If the optimal value is positive for none of these, then we conclude that $\tau_{a}$ is an ADT for $\mathcal{A}$. By adding extra variables and constraints we are able to formulate a single MILP that maximizes the extra switches over all cycles with $K$ or less mode switches, but for simplicity of presentation, we discuss $\operatorname{sf} \operatorname{MOPT}\left(\tau_{a}\right)$ instead of this latter formulation. The following are the decision variables for $\operatorname{MOPT}\left(K, \tau_{a}\right)$; the objective function and the constraints are shown in Figure 5.

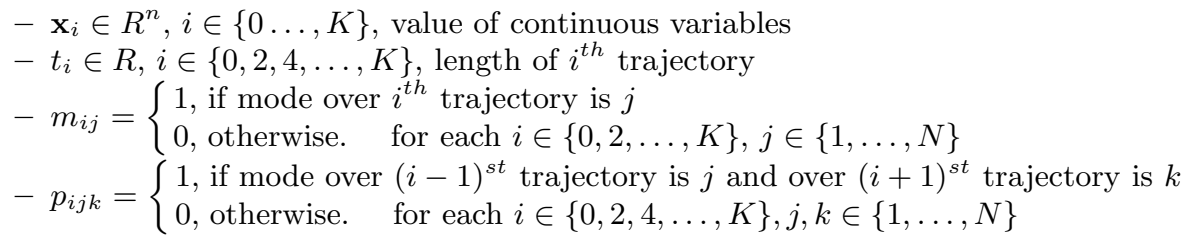

In $\operatorname{MOPT}\left(K, \tau_{a}\right)$, an execution fragment with $K$ mode switches is represented as a sequence $\mathbf{x}_{0}, \mathbf{x}_{1}, \ldots, \mathbf{x}_{K}$ of $K$ valuations for the continuous variables. For each even $i, \mathbf{x}_{i}$ goes to $\mathbf{x}_{i+1}$ by a trajectory of length $t_{i}$. If this trajectory is in mode $j$, for some $j \in\{1, \ldots, N\}$, then $m_{i j}=1$, else $m_{i j}=0$. For each odd $i, \mathbf{x}_{i}$ goes to $\mathbf{x}_{i+1}$ by a discrete transition. If this transition is from mode $j$ to mode $k$, for some $j, k \in\{1, \ldots, N\}$, then $p_{i j k}=1$, else $p_{i j k}=0$. These constraints are specified by Equation (11) in Figure 5. For each odd $i$, Constraints (13) and (14) ensure that $\left(\mathbf{x}_{i}, \operatorname{switch}(j, k), \mathbf{x}_{i+1}\right)$ is a valid mode switching transition. These constraints reduce to the inequalities $G[j, k] \mathbf{x}_{i} \leq g[j, k]$ and $R[j, k] \mathbf{x}_{i+1} \leq r[j, k]$ which correspond to the guard and the initialization conditions on the pre- and the post-state of the transition. For each even $i, \mathbf{x}_{i}$ evolves to $\mathbf{x}_{i+1}$ through a trajectory in some mode, say $j$. Constraint (15) ensures that $\mathbf{x}_{i}$ satisfies the invariant of mode $j$ described by the inequality $A[j] \mathbf{x}_{i} \leq a[j]$. An identical constraint for $\mathbf{x}_{i+1}$ is written by replacing $\mathbf{x}_{i}$ with $\mathbf{x}_{i+1}$ in (15). Since the differential equations have constant right hand sides and the invariants describe pohyhedra in $R^{n}$, the above conditions ensure that all the intermediate states in the trajectory satisfy the mode invariant. Equation (16) ensures that, for each even $i$, $\mathbf{x}_{i}$ evolves to $\mathbf{x}_{i+1}$ in $t_{i}$ time according to the differential equation $d(\mathbf{x})=c[j]$.

Some of these constrains involve nonlinear terms. For example, $m_{i j} \mathbf{x}_{i}$ in (15) is the product of real variable $\mathbf{x}_{i}$ and boolean variable $m_{i j}$. Using the "big M" method [16] we can linearize this equation by replacing $m_{i j} \mathbf{x}_{i}$ with $\mathbf{y}_{i}$, and adding the following linear inequalities: $\mathbf{y}_{i} \geq m_{i j} \delta, \mathbf{y}_{i} \leq m_{i j} \Delta, \mathbf{y}_{i} \leq \mathbf{x}_{i}-\left(1-m_{i j}\right) \delta$, and $\mathbf{y}_{i} \geq \mathbf{x}_{i}-\left(1-m_{i j}\right) \Delta$, where $\delta$ and $\Delta$ are the lower and upper bounds on the values of $\mathbf{x}_{i}$. 
Objective function: $S_{\tau_{a}}: \frac{K}{2}-\frac{1}{\tau_{a}} \sum_{i=0,2, \ldots}^{K} t_{i}$
Mode: $\forall i \in\{0,2, \ldots, K\}, \sum_{j=1}^{N} m_{i j}=1$ and $\forall i \in\{1,3, \ldots, K-1\}, \sum_{j=1}^{N} \sum_{k=1}^{N} p_{i j k}=1$

Cycle: $\mathbf{x}_{0}=\mathbf{x}_{K}$ and $\forall j \in\{1, \ldots, N\}, m_{0 j}=m_{K j}$

Preconds: $\forall i \in\{1,3, \ldots, K-1\}, \sum_{j=1}^{N} \sum_{k=1}^{N} G[j, k] . p_{i j k} \cdot \mathbf{x}_{i} \leq \sum_{j=1}^{N} \sum_{k=1}^{N} p_{i j k} . g[j, k]$

Initialize: $\forall i \in\{1,3, \ldots, K-1\}, \sum_{j=1}^{N} \sum_{k=1}^{N} R[j, k] \cdot p_{i j k} \cdot \mathbf{x}_{i+1} \leq \sum_{j=1}^{N} \sum_{k=1}^{N} p_{i j k} \cdot r[j, k]$

Invariants: $\forall i \in\{0,2, \ldots, K\}, \quad \sum_{j=1}^{N} A[j] \cdot m_{i j} \cdot \mathbf{x}_{i} \leq \sum_{j=1}^{N} m_{i j} \cdot a[j]$

Evolve: $\forall i \in\{0,2 \ldots, K\}, \quad \mathbf{x}_{i+1}=\mathbf{x}_{i}+\sum_{j=1}^{N} c[j] \cdot m_{i j} \cdot t_{i}$

Fig. 5. The objective function and the linear and integral constraints for $\operatorname{MOPT}\left(K, \tau_{a}\right)$

Example 2: Thermostat. We use the MILP technique together with switching simulation relations to verify the ADT of a thermostat with nondeterministic switches. The thermostat SHA $\mathcal{A}$ (see Figure 6 Left) has two modes $l_{0}, l_{1}$, two continuous variables $x$ and $z$, and real parameters $h, K, \theta_{1}, \theta_{2}, \theta_{3}, \theta_{4}$, where $0<\theta_{1}<\theta_{2}<\theta_{3}<\theta_{4}<h$. In $l_{0}$ mode the heater is off and the temperature $x$ decreases according to the differential equation $d(x)=-K x$. While the temperature $x$ is between $\theta_{2}$ and $\theta_{1}$, the on action must occur. As a result of which the mode changes to $l_{1}$. In mode $l_{1}$, the heater is on and the $x$ rises according to the $d(x)=K(h-x)$, and while $x$ is between $\theta_{3}$ and $\theta_{4}$, the off action must occur. The continuous variable $z$ measures the total time spent in mode $l_{1}$

The thermostat SHA $\mathcal{A}$ is neither initialized nor rectangular; however, there is a rectangular initialized SHA $\mathcal{B}$, such that $\mathcal{B} \geq_{A D T} \mathcal{A}$. Consider the SHA $\mathcal{B}$ of Figure 6 (Right) with parameters $L_{0}$ and $L_{1}$. Automaton $\mathcal{B}$ has a reset timer $r$ and two modes $l_{0}$ and $l_{1}$, in each of which $r$ increases at a unit rate. When $r$ reaches $L_{i}$ in mode $l_{i}$, a switch to the other mode may occur and if it does then $r$ is set to zero. We define a relation $\mathcal{R}$ on the state spaces of $\mathcal{A}$ an $\mathcal{B}$ such that with appropriately chosen values of $L_{0}$ and $L_{1}, \mathcal{B}$ captures the fastest switching behavior of $\mathcal{A}$.

Definition 8. For any $\mathbf{x} \in Q_{\mathcal{A}}$ and $\mathbf{y} \in Q_{\mathcal{B}}, \mathbf{x} \mathcal{R} \mathbf{y}$ if and only if: (1) $\mathbf{x}\lceil$ mode $=$ $\mathbf{y}\left\lceil\right.$ mode, and (2) if $\mathbf{x}\left\lceil\right.$ mode $=l_{0}$ then $\mathbf{y}\left\lceil r \geq \frac{1}{K} \ln \frac{\theta_{3}}{\mathbf{x}\lceil x}\right.$ else $\mathbf{y}\left\lceil r \geq \frac{1}{K} \ln \left(\frac{h-\theta_{2}}{h-\mathbf{x} \mid x}\right)\right.$.

Lemma 5. If we set $L_{0}=\frac{1}{k} \ln \frac{\theta_{3}}{\theta_{2}}$ and $L_{1}=\frac{1}{k} \ln \frac{h-\theta_{2}}{h-\theta_{3}}$, then the relation $\mathcal{R}$ is a switching simulation from $\mathcal{A}$ to $\mathcal{B}$. 
Proof: The proof is by induction on the length of an execution of $\mathcal{A}$. The base case follows immediately as the start state of $\mathcal{A}$ is related to the start state of $\mathcal{B}$. Next, for the inductive step we show by cases that given any state $\mathbf{x} \in Q_{\mathcal{A}}, \mathbf{y} \in Q_{\mathcal{B}}, \mathbf{x} \mathcal{R} \mathbf{y}$, and an execution fragment $\alpha$ of $\mathcal{A}$ starting from $\mathbf{x}$ and consisting of either a single action or a single trajectory, there exists a corresponding execution fragment $\beta$ of $\mathcal{B}$, starting from $\mathbf{y}$ that satisfies the conditions required for $\mathcal{R}$ to be a switching simulation relation.

1. $\alpha$ is a $\left(\mathbf{x}\right.$, on, $\left.\mathbf{x}^{\prime}\right)$ transition of $\mathcal{A}$.

We choose $\beta$ to be the $\left(\mathbf{y}\right.$, on, $\left.\mathbf{y}^{\prime}\right)$ transition of $\mathcal{B}$. First we show that the action on is enabled at $\mathbf{y}^{\prime}$. Since $\mathbf{x} \mathcal{R} \mathbf{y}$, we know that $\mathbf{y}\left\lceil\right.$ mode $=\mathbf{x}\left\lceil\right.$ mode $=l_{0}$ and further, $\mathbf{y}\left\lceil r \geq \frac{1}{k} \ln \frac{\theta_{3}}{\mathbf{x}\lceil x}\right.$. But we know that, $\mathbf{x}\left\lceil x \leq \theta_{2}\right.$ because the on action of $\mathcal{A}$ is enabled at $\mathbf{x}$. It follows that $\mathbf{y}\left\lceil r \geq \frac{1}{k} \ln \frac{\theta_{3}}{\theta_{2}}=L_{0}\right.$, that is, the on action is enabled at $\mathbf{y}$. Next we show that $v x^{\prime} \mathcal{R} \mathbf{y}^{\prime}$. Its immediate that $\mathbf{x}^{\prime}\left\lceil\right.$ mode $=l_{1}=\mathbf{y}^{\prime}\lceil$ mode. From the transition definition of both the on actions, it follows that $\mathbf{y}^{\prime}\left\lceil r=0\right.$ and $\mathbf{x}^{\prime}\left\lceil x=\mathbf{x}\left\lceil x \leq \theta_{2}\right.\right.$ and $\ln \frac{h-\theta_{2}}{h-\mathbf{x}^{\prime}\lceil x} \leq 0$. Therefore, $\mathbf{y}^{\prime}\left\lceil r \leq \frac{1}{K} \ln \frac{h-\theta_{2}}{h-\mathbf{x}^{\prime} \mid x}\right.$.

2. $\alpha$ is a $\left(\mathbf{x}\right.$, off, $\left.\mathbf{x}^{\prime}\right)$ transition of $\mathcal{A}$.

We choose $\beta$ to be the $\left(\mathbf{y}\right.$, on, $\left.\mathbf{y}^{\prime}\right)$ transition of $\mathcal{B}$ and the rest of the proof is similar to that of case 1 .

3. $\alpha$ is a single trajectory $\tau$ of $\mathcal{A}$ such that $(\tau \downarrow$ mode $)(0)=l_{0}$. We choose $\beta$ to be a trajectory $\tau^{\prime}$ of $\mathcal{B}$ defined as follows:

$$
\forall t \in \tau^{\prime} . \operatorname{dom},\left(\tau^{\prime} \downarrow \operatorname{mode}\right)(t)=l_{0} \text { and }\left(\tau^{\prime} \downarrow r\right)(t)=\left(\tau^{\prime} \downarrow r\right)(0)+t
$$

It follows immediately that $\tau^{\prime}$ is a valid trajectory for $\mathcal{B}$ because it satisfies the the differential equation $d(r))=1$ and the mode remains constant.

4. $\alpha$ is a single trajectory $\tau$ of $\mathcal{A}$ such that $(\tau \downarrow$ mode $)(0)=l_{1}$. The proof for this case is the same as that of the previous case.

Lemma 5 implies that $\mathcal{B} \geq_{A D T} \mathcal{A}$, and so, for any $\tau_{a}>0$ if $\tau_{a}$ is an ADT for $\mathcal{B}$ then $\tau_{a}$ is also an ADT for $\mathcal{A}$. Also, $\mathcal{B}$ is rectangular and initialized, so we can use Theorem 4 and MILP technique to check any ADT property of $\mathcal{B}$.

We formulated the $\operatorname{MOPT}\left(K, \tau_{a}\right)$ for automaton $\mathcal{B}$ and used the GNU Linear Programming Kit [1] to solve it. Solving for $K=4, L_{0}=40, L_{1}=15$, and $\tau_{a}=$ $25,27,28$, we get optimal costs $-0.4,-4.358 E^{-13}(\approx 0)$ and 0.071 , respectively. We conclude that the ADT of $\mathcal{B}$ is $\geq 25, \geq 27$, and $<28$. Since $\mathcal{B} \geq_{A D T} \mathcal{A}$, we conclude that the ADT of the thermostat is no less than 27 .

Remark 2. For finding counterexample execution fragments of proposed ADT properties, the MILP approach can be applied to non-initialized rectangular SHA as well. In such applications, however, the necessity part of Theorem 4 will not hold and from the failure to find a counterexample alone we cannot conclude that the automaton satisfies the ADT property in question. 


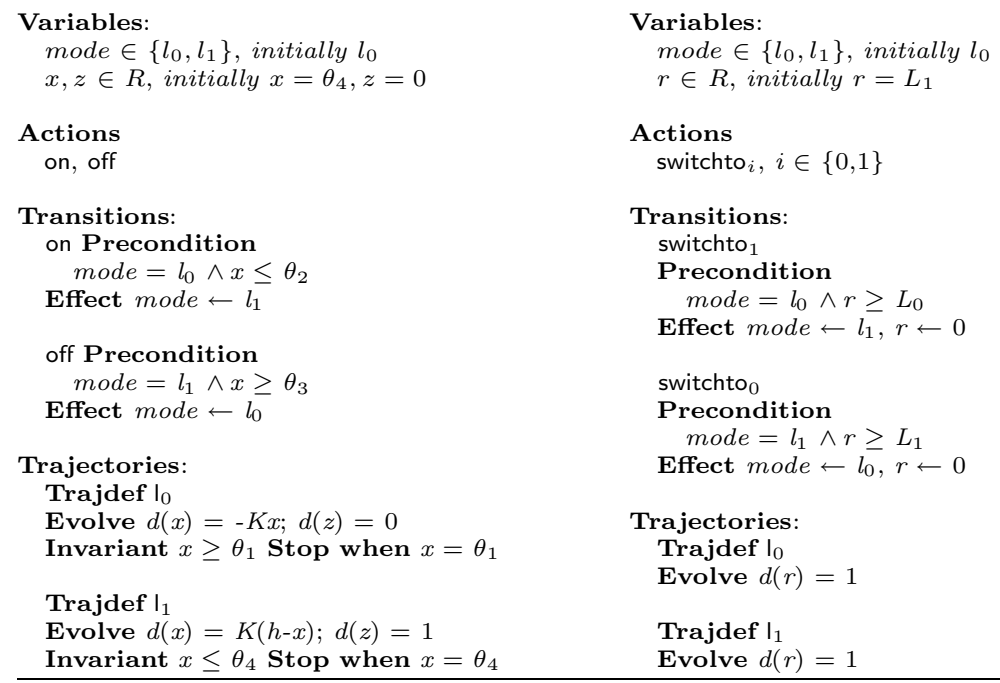

Fig. 6. Left:Thermostat SHA $\mathcal{A}$ with parameters $\theta_{1}, \theta_{2}, \theta_{3}, \theta_{4}, K$, and $h$. Right: Rectangular SHA $\mathcal{B}$ with parameters $L_{0}, L_{1}$.

Conclusions. We have presented optimization-based methods for automatically verifying Average Dwell Time (ADT) properties of certain classes of hybrid systems, which provides a tool for proving (uniform) stability. We have also defined equivalence of hybrid systems with respect to ADT and have presented a simulation relation-based method for proving these equivalence relationships. The proposed methods have been applied to verify ADT of a linear, scale-independent hysteresis switch and a nondeterministic thermostat.

In this paper we examined internal stability only; however, the input and output variables of HIOA make the framework suitable for studying input-output properties of hybrid systems. Another direction of future research is to extend the ADT verification technique to probabilistic hybrid systems.

Acknowledgments. We would like to thank Debasish Chatterjee for carefully reading the manuscript and for his comments.

\section{References}

1. GLPK - GNU linear programming kit. http://www.gnu.org/directory/libs/glpk.html.

2. M. Branicky. Multiple Lyapunov Functions and other analysis tools for switched and hybrid systems. IEEE Transactions on Automatic Control, 43:475-482, 1998.

3. T. H. Cormen, Charles E. Leiserson, and Ronald L. Rivest. Introduction to Algorithms. MIT Press/McGraw-Hill, 1990.

4. C. Heitmeyer and N. Lynch. The generalized railroad crossing: A case study in formal verification of real-time system. In Proceedings of the 15th IEEE Real-Time Systems Symposium, 1994. 
5. J.P. Hespanha, D. Liberzon, and A.S. Morse. Hysteresis-based switching algorithms for supervisory control of uncertain systems. Automatica, 39:263-272, 2003.

6. J.P. Hespanha and A. Morse. Stability of switched systems with average dwelltime. In Proceedings of 38th IEEE Conference on Decision and Control, pages 2655-2660, 1999.

7. D. Kaynar, N. A. Lynch, R. Segala, and F. Vaandrager. The theory of timed I/O automata. Technical Report MIT/LCS/TR-917a, MIT Laboratory for Computer Science, 2004. Available at http://theory.lcs.mit.edu/tds/reflist.html.

8. D. K. Kaynar, N. A. Lynch, R. Segala, and F. Vaandrager. Timed I/O automata: A mathematical framework for modeling and analyzing real-time system. In RTSS 2003: The 24th IEEE International Real-Time Systems Symposium, Cancun,Mexico, December 2003.

9. D. Liberzon. Switching in Systems and Control. Systems and Control: Foundations and Applications. Birkhauser, Boston, June 2003.

10. C. Livadas, J. Lygeros, and N. A. Lynch. High-level modeling and analysis of TCAS. In Proceedings of the 20th IEEE Real-Time Systems Symposium, 1999.

11. N. A. Lynch, R. Segala, and F. Vaandrager. Hybrid I/O automata. Information and Computation, 185(1):105-157, August 2003.

12. S. Mitra and D. Liberzon. Stability of hybrid automata with average dwell time: an invariant approach. In Proceedings of the 43rd IEEE Conference on Decision and Control, 2004.

13. S. Mitra, Y. Wang, N. A. Lynch, and E. Feron. Safety verification of model helicopter controller using hybrid Input/Output automata. In HSCC'03, Hybrid System: Computation and Control, 2003.

14. A. S. Morse. Supervisory control of families of linear set-point controllers, part 1: exact matching. IEEE Transactions on Automatic Control, 41:1413-1431, 1996.

15. A. van der Schaft and H. Schumacher. An Introduction to Hybrid Dynamical Systems. Springer, London, 2000.

16. H.P. Williams. Model building in mathematical programming. J. Wiley, New York, 1990. third edition. 